\title{
Five-Factor Inventory of Intrinsic Motivations to Gameplay (IMG)
}

\author{
Jukka Vahlo \\ Game Research Lab \& Gamification Group \\ Tampere University \& University of Turku \\ jukka.vahlo@uta.fi
}

\author{
Juho Hamari \\ Gamification Group \\ Tampere University \& University of Turku \\ juho.hamari@tut.fi
}

\begin{abstract}
In this study, we develop and validate Intrinsic Motivations to Gameplay (IMG) inventory. In Study 1, psychometric properties of a preliminary 10-item version of IMG were investigated by employing an online survey data collected among Finnish and Danish population ( $N=2,205)$. In Study 2, a 23-item version of IMG was developed based on further interview data and survey data collected among Canadian population $(N=1,322)$. The 23-item version of IMG revealed five factors of intrinsic motivations for gameplay: Relatedness, Autonomy, Competence, Immersion, and Fun. In Study 3, a third survey was conducted among Finnish and Japanese participants $(N=2,057)$ to design a SelfDetermination theory (SDT) informed confirmatory factor analysis (CFA). The CFA validated a 15-item version of IMG inventory, which can be utilized widely in studies on digital gaming and gamification to better understand player preferences.
\end{abstract}

\section{Introduction}

Understanding play, players and why people play games and participate in different kinds of gameful and playful activities has been a major vein of research in the overlap of game studies, media psychology, computer science and information systems science. Currently, player research has mainly divided under three broad categories: 1) investigation of player preferences (e.g. $[1][17][22][43][47][51]), 2)$ the gratification that players derive (e.g. [16]), and 3) the demographic factors of players and their avatars (e.g. [13][14][15][24][25]).

Being able to scrutinize the aspects of motivating play is important for designing games and gamified systems since the main purpose of gameful interaction is essentially to provide motivational affordances ([24]). Furthermore, better knowledge of what motivates to play can be utilized in constructing player types and predicting patterns in players' habitual game choices ([48][49]).

While the literature on player motivations is a rapidly growing area of research, many popular models used in measuring motivations to play have shortcomings. Several widely utilized models are genre-specific (e.g. [51][52]), or lack validation with behavioral data and cross-cultural data. (See [6][7] [21]). Because of these shortcomings, existing models cannot be utilized widely in measuring general reasons for intrinsically motivating game play. Conducting e.g. comparative studies on cultural differences in motivations to play necessitates developing a new scale which 1) focuses on prevalent reasons to play digital games, and which 2) is properly validated across cultures.

The purpose of this study is to develop and validate a psychometrically valid short inventory for measuring intrinsically motivating gameplay. We begin the article by relating our motivations to play approach to prior research on player preferences and behavior. We proceed then to conduct a series of exploratory factor analyses (EFA) with representative survey samples from Finland, Denmark, and Canada. This will be followed by an item screening process and discussions of the theoretical implications of the EFAs. We continue then to conduct a CFA with combined survey data from Finland and Japan $(\mathrm{N}=2,057)$. The article will be concluded with a discussion on how the results of this study may inform future game research.

\section{Theoretical Background}

In order for a person to act, she needs to be motivated by something. In the motivation theory, it is often argued that motivations can be placed on a continuum from extrinsic to intrinsic ([37]). In extrinsically motivating activities, a person engages with an activity primarily because of external pressure toward a specific instrumental outcome. In contrast to this, activities which are intrinsically motivating are experienced as inherently enjoyable 
and satisfying. According to Self-Determination theory (SDT), intrinsically motivating activities are frequently perceived as fun and entertaining. ([8][37]) This is largely because the subject experiences that her needs and motivations align with the requirements and characteristics of the situation at hand ([9][12]) Thus, a central objective for designers of both games and other gamified systems is to enable experiences that are intrinsically motivating.

Out of the overall body of player research, the player preference literature can be further divided into four main categories: motivation to play, player behavior, gaming intensity, and gameplay type preferences ([46][48]).

Motivations to play literature (e.g. $[3][6][16][26][34][40][50][51][52])$ asks why we play games. Motivations to play studies can be divided into general models and contextual models. For example, in contrast to Yee's ([51]) empirical approach on identifying motivations to play online games (contextual approach), Ryan, Rigby and Przybylski ([38]) have argued from a SDT stance that studies on motivations to play should focus on psychological theories of human motivations instead of specific characteristics of gaming situations. This latter general approach would mean that the reasons for our playing are the same as our reasons to engage with any activities. According to SDT, gaming situations are not in that sense unlike everyday life.

Analyzing the general research question of why we play provides us tools for understanding habitual play, but it does not necessarily help us to predict which specific games players purchase ([48]). On the other hand, player behavioral models (e.g. $[1][2][5][30][45])$ are able to analyze what kind of playing styles players adopt during gameplay of a particular game. These models ask how players differ in their behavioral patterns of play.

Gaming intensity approach (e. g. [9][20][22]) shares some qualities with both motivations to play models and player behavior approaches. Studies on gaming intensity ask what mentality and gaming mode a player adopts. Also these studies may focus on either general or contextual aspects of gaming mentalities. The general models aim to distinguish hardcore gamers, committed gamers, regular gamers and casual gamers from each other (see [22]). The contextual models are more interested in studying which technology is available for the player, what kind of play modes (single-player, local co-op, multiplayer) and camera angles the technology affords, and how the current mood of the player affects her game choice and playing behavior ([9]).

Recently, a few studies have proposed that motivations to play models and player behavioral models could be complemented by intermediate models which investigate patterns in players' gameplay type preferences ([17][43][44][47][48]). This field of research covers subjects such as players' preferences in gameplay activity types, gameplay challenge types, and in the interactive, narrative, and audiovisual qualities of a variety of games ([46][47]). The core research question for these models is which games players prefer to play and how these preferences influence their game choice ([47][48]).

It is relevant for the current study to distinguish motivational models, behavioral models, gaming intensity models, and gameplay type preference models from each other. Establishing a distinction between these approaches of player preference research enables us to focus on developing inventory items for studying each aspect of gaming phenomenon both individually and combined. Indeed, a shortcoming for many prior motivations to play inventories is that they mix together general motivations, gameplay type preferences, gaming intensity dimensions, and play style attributes.

For instance, De Grove et al. ([6]) conducted a series of extensive studies to confirm an instrument for measuring individual motives to play digital games. Their social cognitive theory (SCT) based model combines dimensions of habit, moral selfreflection, agency, narrative, escapism, pastime, performance, and social interaction. The model bundles together many elements which indeed can motivate players, but which can also be argued to measure gameplay preferences and gaming intensities rather than motivations. (See also [21])

Because the aim of this article is to develop an inventory for studying the general intrinsic motivations to play, the inventory items of this study are not developed to measure how players prefer to play (behavior), what contextual or sustaining gaming modes they find enjoyable (intensity), or which gameplay activity types, challenge types and narrative/interactive/audiovisual qualities they find appealing. By focusing on general motivations to play, the inventory developed in this article is applicable for studying all kinds of gameplay experiences, regardless of the content of the game.

\section{Study 1: Exploratory Factor Analysis}

\subsection{Survey Participants}

In Study 1, a survey was conducted for investigating players' gameplay type preferences, playing style preferences, gaming modes, and intrinsic motivations to play. We recruited a total of 
2,594 respondents from Finland and Denmark in cooperation with an international market research company to obtain a representative sample of the populations from both countries (ages 12-70). The market research company cleaned the data by analyzing participants' response time and by removing cases that replied to the survey too quickly. Furthermore, we cleaned the data from participants who showed content nonresponsivity by responding similarly to every question ([29]) and from those who indicated that they were not motivated to play digital games at all. As a result, a total of 389 participants were removed from the cleaned sample. The final sample included in the EFA consisted of 2,205 respondents (mean age 37.7, 52.8\% men)

\subsection{Materials and procedure}

The survey participants were instructed to think about their reasons to play and specify how important (5-Likert, 1 = completely unimportant, $5=$ very important) a total of 12 motivations were for their habit of playing digital games. The survey included also a gameplay activity type and gameplay challenge type preference inventory, an inventory on preferred game qualities and elements, an inventory on player behavior preferences, and an inventory on favored gaming modes and intensity. The survey also included questions regarding participants' age, gender, income, expenditure on games and weekly play time. The data was collected with a web-based survey tool, and it took about 20 minutes to take the whole survey with a computer or a mobile phone.

The twelve items of the initial scale were developed based on a literature review of prior motivations to play models (e.g.
$[3][26][34][40][51][52])$. A focus group meeting with two game designers and game design course participants was then organized to discuss about the findings of the literature review, and to select wordings for inventory items. The scale was then piloted in a study of 50 university students who had an opportunity to suggest new items to the inventory. The purpose of this scale was to measure motivations to play with single-item measures. However, an EFA was also conducted to investigate the possible underlying latent structures of the scale.

\subsection{Results}

The number of factors to be extracted for the 12item inventory was identified by conducting a parallel analysis ([18]). We first made an EFA by using statistical software Stata 14.2 on the data which was followed by parallel analysis (PA) test, which generates an artificial data set for identifying the correct number of factors. The PA test suggested that four factors were to be extracted. The Kaiser-MeyerOlkin (KMO) test was utilized to measure sampling adequacy for conducting a factor analysis. The KMO value was good (0.863).

An EFA with four factors was conducted by using principal axis factors and promax rotation. Promax rotation was selected instead of varimax rotation, since the former allows factors to correlate with each other and does not assume them to be orthogonal to each other. ([28]) This was an informed decision, since it is plausible to assume that a person who enjoys playing digital games is motivated by several factors instead of just one.

Table 1. An EFA of the 10-item motivations to play inventory. Factor loadings and uniqueness for the items.

\begin{tabular}{|c|c|c|c|c|c|c|}
\hline & Motivations & 1 & 2 & 3 & 4 & Uniq. \\
\hline 1 & I play with my family and friends because of their company & 0.644 & & & & 0.564 \\
\hline 2 & I play to relax & & & & 0.688 & 0.511 \\
\hline 3 & I play for the fun of playing & & & & 0.676 & 0.408 \\
\hline 4 & I play because I am interested in different games & & 0.670 & & & 0.346 \\
\hline 5 & I play because I want to get immersed in games & & 0.675 & & & 0.345 \\
\hline 6 & I play online because of the other players & 0.707 & & & & 0.416 \\
\hline 7 & I play because my friends play & 0.751 & & & & 0.402 \\
\hline 8 & I play because of competition & 0.414 & & 0.429 & & 0.510 \\
\hline 9 & I play so that I can get feelings of achievement and success & & & 0.603 & & 0.443 \\
\hline 10 & I play to face challenges and to develop my skills & & & 0.561 & & 0.458 \\
\hline
\end{tabular}

We used the factor loading $>.40$ as a criterion to determine whether an item loaded on a factor. In the first solution, two items "I play to kill time" and "I play to avoid anxiety" did not load on any factor. We removed these two items and conducted another PA test to identify the number of factors. The PA test suggested still that four factors were to be extracted. In the second solution all items loaded on a factor. The item "I play because of competition" crossloaded on the factors 1 and 3. Only two items loaded 
on factors 2, 3 and 4. Since at least three items should load on a factor in order for it to be identified ([4]), we do not report Cronbach's alphas for this preliminary study.

Items 1, 6 and 7 loaded on the first factor. These three items indicate that a player is motivated to play because of the social element in a gaming situations. Items 3 and 4, which both loaded on factor 2, refer to inherent interest towards gaming and a will to experience immersion in gameplay. Items which portrayed challenge and achievement loaded on the third factor. Finally, items which emphasize motivational qualities of relaxation and fun loaded on the factor 4 .

These initial results are largely in line with prior literature on motivations to play. Yet it was a bit surprising that fun and relaxation loaded on a same factor. Since playing for fun is often considered as a banal statement, we presumed that the item 3 would not load on any factor but instead show several crossloadings. However, both the items of "I play to kill time" and "I play to avoid anxiety" which did not load on any factor can be interpreted to more extrinsically motivated reasons to play than the ten other items.

\section{Study 2: Developing the Inventory}

We continued to develop new items to the inventory by conducting 32 interviews with gamers who had participated in the survey of Study 1 . The interviewees were selected to represent different aspects of gaming preferences. The youngest interviewee was 19 years old and the oldest 49 . A total of 18 of the interviewees were female players. Interviews lasted from 45 minutes to 110 minutes, and they were recorded with an audio recording device and later transcribed by a company which provides transcription services for academic purposes.

When asked to describe with their own words the reasons for their hobby of playing digital games, most replied according to the preliminary four-factor model (Table 1). The typical first reaction to this question was simply: "because it's fun". But after a few follow-up questions, most players described their motivations to play in a much more detailed way. In addition to the four preliminary factors (Table 1), several players told that a main driver for their playing was an experience of freedom, being able act independently, and the possibility to make a difference in the gameworld.

\subsection{Survey Participants}

Based on a content analysis of the interview material and also on open-ended feedback from the Study 1, a 25-item motivations to play scale was developed for a follow-up survey to examine players' motivations to play digital games. A total of 1,500 participants were recruited from Canada (ages 1865). Participants were asked to report in the beginning of the survey how interested they were in playing digital games (5-Likert, $1=$ not at all, $5=$ very interested). If a participant replied that she was not at all interested in playing games, she was thanked for participating and instructed to the end of the survey. This was done because analyzing motivations is sensible only if a person is at least a little bit interested in the activity.

The sample was cleaned according to the procedure reported in Study 1. A data cleaning procedure is generally encouraged for factor analytical studies aiming for scale development ([29]). As a result, a total of 178 participants were removed from the sample. The final sample consisted of 1,322 respondents $(50.4 \%$ men, mean age 41.4 years).

\subsection{Materials and procedure}

The survey of Study 2 included a refined 25 -item motivations to play inventory (5-Likert, $1=$ completely unimportant, $5=$ very important). The survey included also a gameplay activity type inventory, a gameplay challenge type preference inventory, an inventory on preferred game qualities, and a set of demographic questions. The data was collected via a web-based survey tool. The survey was translated to English and French and backtranslated to Finnish.

The 25 items were developed based on the results of Study 1 and a content analysis of the 32 interviews. Since the interviews supported all of the four preliminary factors, new items were designed by keeping this result in mind. Also, five new items were fashioned based on interviewees' reflections on how being free and independent could form yet another motivational factor. ([46])

\subsection{Results}

A PA test was made for identifying the number of underlying factors in the 25 -item inventory. The PA test suggested a five-factor structure, and thus we proceeded to conduct an EFA (principal axis factors, promax rotation). KMO test value (0.951) indicated that conducting a factor analysis was adequate. 
We utilized the factor loading $>.50$ as a criterion to determine whether an item loaded on a factor. In the first solution, the items: "I play to win my opponents or enemies in the game" and "I play to get experiences of being successful" did not load on any factor. We excluded these items and conducted another PA test, which suggested again a five-factor structure. This time all items loaded on a factor with a loading $>.50$ without cross-loadings (Table 2).

The five items which loaded on the first factor indicate that the player is motivated by being part of the events and life stories that take place in the gameworld. She also enjoys that gameplay induces deep emotions. We call this factor Immersion. The five items which loaded on the second factor indicate a motivation to play because games enable selfrealization, independent action and expressions of free will. This factor is Autonomy.

Three items loaded on the third factor, which we label Competence. A person motivated by this factor plays because she enjoys mastering her skills by overcoming challenges, making in-game progress and achieving goals. The five items which loaded on the fourth factor denote motivations towards experiences that are entertaining, fun, pleasurable, and relaxing. We name this factor simply Fun. Finally, the five items which loaded on the fifth factor are based on social connectedness in a gameplay situation. A person plays because she finds shared experiences gratifying. We call this factor Relatedness.

Table 2. An EFA of the 23-item motivations to play inventory. Factor loadings and descriptive statistics for the scale.

\begin{tabular}{|c|c|c|c|c|c|c|c|}
\hline & Motivations & 1 & 2 & 3 & 4 & 5 & Uniq. \\
\hline 1 & I play online games because I enjoy interacting with others & & & & & 0.587 & 0.394 \\
\hline 2 & I play because also my friends play & & & & & 0.776 & 0.332 \\
\hline 3 & I play with my family because of the company & & & & & 0.824 & 0.330 \\
\hline 4 & I play with my friends because of the company & & & & & 0.889 & 0.214 \\
\hline 5 & I play because I enjoy especially playing together & & & & & 0.823 & 0.250 \\
\hline 6 & I play because of the challenge & & & 0.693 & & & 0.421 \\
\hline 7 & I play to master my skills and to win myself & & & 0.796 & & & 0.335 \\
\hline 8 & I play to make progress and to achieve objectives & & & 0.620 & & & 0.392 \\
\hline 9 & I play because I want to immerse myself in games & 0.644 & & & & & 0.367 \\
\hline 10 & I play because I want to identify with the game characters & 0.810 & & & & & 0.222 \\
\hline 11 & I play because the game's story and its mysteries fascinate me & 0.736 & & & & & 0.397 \\
\hline 12 & I play because game events bring about emotions & 0.822 & & & & & 0.253 \\
\hline 13 & I play because I want to be part of the gameworld and its events & 0.788 & & & & & 0.243 \\
\hline 14 & I play because it is fun & & & & 0.804 & & 0.383 \\
\hline 15 & I play because playing games is relaxing & & & & 0.719 & & 0.439 \\
\hline 16 & I play because games are entertaining & & & & 0.902 & & 0.197 \\
\hline 17 & I play because games are enjoyable & & & & 0.915 & & 0.231 \\
\hline 18 & I play because playing makes me feel good & & & & 0.588 & & 0.400 \\
\hline 19 & I play because in games I can be independent & & 0.777 & & & & 0.338 \\
\hline 20 & I play because in games I can make my own decisions & & 0.796 & & & & 0.306 \\
\hline 21 & I play because in games I can make a difference with my & & 0.736 & & & & 0.213 \\
\hline 22 & I play because in games I can $n$ & & 0.741 & & & & 0.206 \\
\hline 23 & I play because in games I can realize $n$ & & 0.549 & & & & 0.273 \\
\hline & $\mathrm{Me}$ & 2.881 & 3.143 & 3.378 & 4.035 & 2.797 & \\
\hline & Star & 1.140 & 1.104 & 0.984 & 0.821 & 1.030 & \\
\hline & Cronbach's Alpha & 0.918 & 0.916 & 0.836 & 0.901 & 0.916 & \\
\hline
\end{tabular}

We conducted an additional EFA with a subsample of survey participants who reported that they spent more time playing free-to-play and premium mobile games than PC and console games. Of the sample collected from Canada, a total of 452 respondents were mobile gamers $(34 \%$ of the sample, mean age 39.1 years, $33.6 \%$ men). The PA test for 23 -items scale suggested that five factors were to be extracted. The results were highly similar to those of the whole Canadian sample. The only exception was that item 23 loaded on the Immersion factor instead of Autonomy.

We furthermore investigated if the exploratory five-factor structure could be identified with a subsample of participants who reported to play online games with PC, console or mobile phones more than non-mobile games. This subsample consisted of 401 participants $(30 \%$ of the sample, mean age 38.7 years, $63.3 \%$ men). The PA test suggested again a five-factor structure. In this EFA, the items 1 and 25 
did not load on any of the five factors, but otherwise the results were identical to the previous EFAs.

The results of the five-factor structure revealed in this study can be interpreted to be congenial with the psychological Self-Determination theory (SDT) of human needs and motivations (e.g. [8][34][37][38]). SDT argues that autonomy, competence, and relatedness are the three basic human needs which motivate us to engage with worldly activities.

In SDT, autonomy refers to the need and "pleasure of being the cause," as Piaget ([32]) put it. It is the willingness and volition to engage with an activity in which a person experiences freedom of choice and gets clear feedback. Competence is the human need for putting one's skills in use to overcome challenges, to learn new skills and master the tasks at hand. And relatedness is the need for being socially connected to close others ([38]).

The factors 2, 3 and 5 (Table 2) are similar to the three basic human needs of SDT. However, also the factors of Fun and Immersion are both congenial with the macro-theory of SDT. According to SDT, situations in which a person experiences need satisfaction are intrinsically motivating. In contrast to this, extrinsically motivating activities diminish the subject's perception of herself as being the locus of causality and control ([8]). An intrinsically motivating activity is inherently enjoyable, satisfying and typically described as fun. ([37])

Furthermore, SDT studies have revealed that satisfaction of competence, autonomy, and relatedness in gameplay experiences increase players' sense of immersion across game contents, elements and genres ([33][38]). Indeed, Przybylski et al. ([34]) summarize: "When players have their needs satisfied within the game, they are more phenomenologically embedded in the emotional, physical, and narrative elements of the game world".

The EFA-based model identified in Study 2 can thus be argued to be compatible with SDT, although it was not constructed from this theoretical framework. The three factors of Autonomy, Competence, and Relatedness are qualitatively similar to the SDT needs, and both Fun and Immersion are also supported by the SDT literature. However, the SDT literature suggests that Fun and Immersion are both indicators of intrinsically gratifying experiences which emerge when the SDT needs are fulfilled. Indeed, SDT needs are usually considered to be precursors of both fun and immersion.

From a SDT-inclined theoretical stance it would have been plausible that Fun and Immersion would not have not formed their own motivational factorsor that the items which loaded on Fun and Immersion would have shown relatively high cross-loadings on the three factors similar to the SDT needs. The fact that this did not happen indicates that players do perceive fun and immersion to be independent types of experience, different from experiences of being competent, autonomous and related to others.

\section{Study 3: Confirmatory Factor Analysis}

A CFA for the 23-item Intrinsic Motivations to Gameplay (IMG) inventory was designed. The CFA was planned to be conducted as a comparative study between countries other than Canada. It is important to test validate of an inventory with cross-cultural data, especially in the case of digital games, because reasons to play differ between countries ([35]). Also, wordings of the inventory items may be understood differently across cultures ([7]).

The CFA was based on the results of the two EFAs reported (Study 1, Study 2) and on the observations that the five-factor structure of Study 2 shared qualitative similarities with SDT-although the results indicated that players consider Fun and Immersion to be independent reasons to play, and not something which could be reduced to the experiences of Competence, Relatedness, and Autonomy.

By conducting a CFA, we asked whether the EFA-screened 23 items could be validated psychometrically as indicators of the latent factor they were developed and designed to measure. Another objective for this study was to shorten the inventory to make it more applicable for research.

\subsection{Participants}

A total of 2,553 respondents (ages 18-65) were obtained from Finland and Japan. The sample was collected according to a similar procedure than those reported in Study 1 and Study 2.

\section{2 Materials and Procedure}

The IMG inventory included in Study 3 was kept identical to the survey conducted in Canada. The English version of the survey was translated to Japanese and back-translated to English. The samples from Japan and Finland were cleaned by a similar procedure than the Canada sample in Study 2. As a result, a total of 174 participants were excluded from the sample collected in Finland, and 322 participants from the Japan sample. The final sample from Finland included in the CFA had 879 respondents (49.5\% men, mean age 41.5 years). Cleaning the Japan sample resulted in 1,178 respondents $(55.0 \%$ 
men, mean age 41.3 years). The combined sample from Japan and Finland consisted of 2,057 participants ( $52.6 \%$ men, mean age 42.2 years).

\subsection{Results}

To conduct a CFA on the five-factor structure of hypothesized intrinsic motivations to gameplay, we constructed 3 -item scales for each of the five factors. This was done because the objective was to validate a short version of the inventory, which could then be more easily included in game research surveys. Three items for a factor is considered to be sufficient for validating a latent construct ([4]).

The 15 items which were included in the CFA were selected based on the results from Study 2. Each item fulfilled the following criteria: 1) the item showed a strong loading on the corresponding factor $(>.50), 2)$ the discrepancy value between the primary and the secondary loading was high $(>.30) ; 3)$ the qualitative aspects the item did not overlap with the other selected two items in a redundant way; and 4) the item did not include words that directly referred to the label of the hypothesized factor. (See [28])

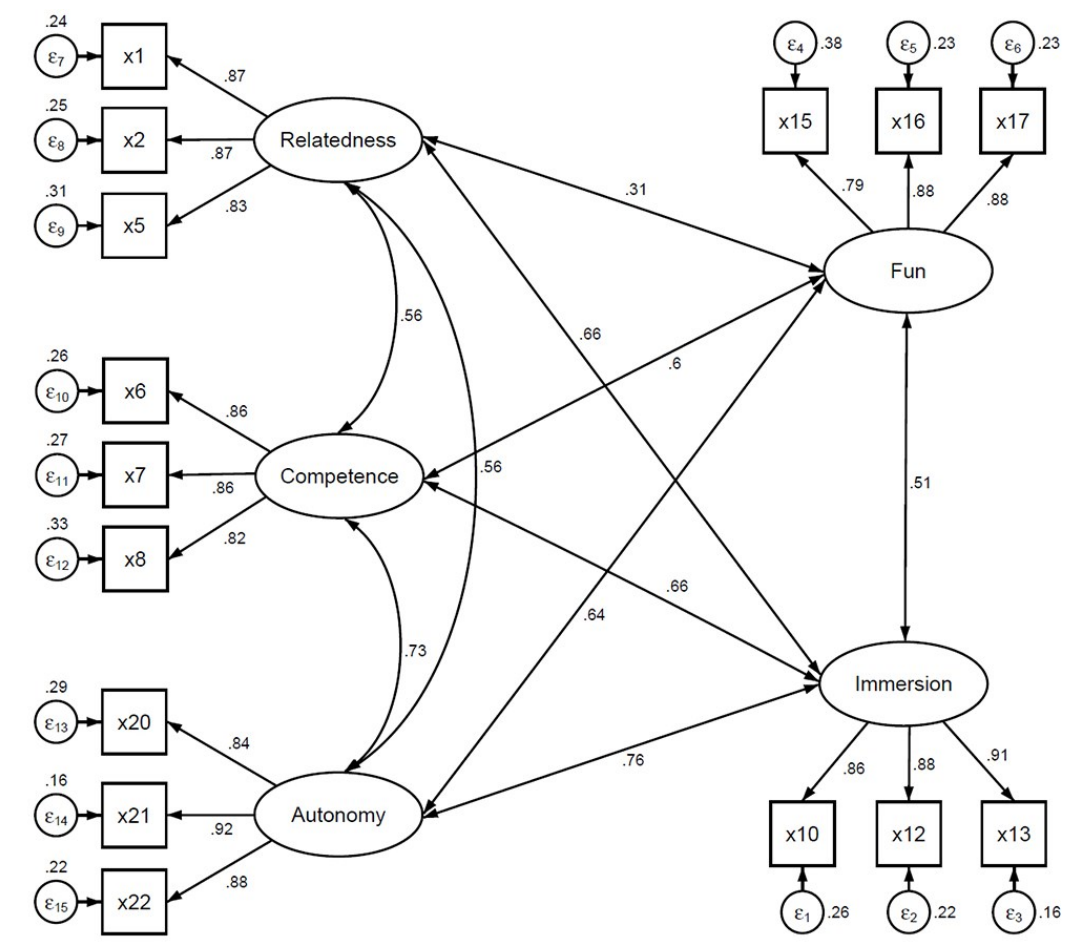

Figure 1. The measurement model reporting confirmatory factor analysis for the 15 -item IMG inventory $(\mathrm{N}=2,057)$. All loadings of the scale are significant on the level $p<0.001$.

Based on this criteria, the items $3,4,9,11,14,18$, 19 , and 23 (Table 2) were excluded from the 15-item version of the IMG. Item 1 was included in the short version because it refers to online gaming and it portrays thus an important aspect not covered by items 2 and 5. Item 19 was removed from the hypothesized Autonomy factor, because of its qualitative similarity with item 20.

The CFA analysis with the combined sample from Finland $(n=879)$ and Japan $(n=1,178)$ was made by structural equation modelling (SEM) by using statistical software Stata 14.2 and maximum likelihood estimation procedure. The measurement model for reporting the CFA is presented in Figure 1. Construct validity for the five factor model was studied by calculating the Tucker Lewis Index (TLI), the comparative fit index (CFI), the root mean squared error of approximation (RMSEA), and the standardized root mean squared residual score (SRMR). The chi square test was not used since it fits poorly to studies with large sample sizes and to models in which correlations are strong ([28][36]).

The goodness-of-fit values for the model we report in Figure 1 were: TLI 0.966, CFI 0.974, RMSEA 0.062, and SRMR 0.034. The value for RMSEA can be regarded acceptable, while the values for the other three indices indicate close fit to the data of 2,057 participants from Japan and Finland. Taken together, the fit indices suggest construct validity for the model. ([4][19][23][27][39]) 
We conducted a composite reliability (CR) test and an average variance extracted analysis (AVE) to study the convergent and discriminant validity of the five factor model. The $\mathrm{CR}$ values for the five factors were: Relatedness (0.893), Competence (0.883), Autonomy (0.912), Fun (0.886), and Immersion (0.916). These results clearly exceed the acceptable value of 0.7 (see [53]).
The AVE test ([10][53]) should be $>.50$ for each latent construct to demonstrate convergent validity. The test is then used to study if the AVE for each construct is higher than the square of the correlation between latent constructs. The results of both the CR test and the AVE test (Table 3) supported convergent and discriminant validity for the 15-item IMG inventory (see [10][11]).

Table 3. The Average Variance Extracted Analysis (AVE) on the five-factor model of the 15-item IMG inventory.

\begin{tabular}{rccccc}
\hline & Relatedness & Competence & Immersion & Fun & Autonomy \\
\hline Relatedness & $\mathbf{0 . 7 8 4}$ & & & & \\
Competence & 0.311 & $\mathbf{0 . 7 2 2}$ & & & \\
Immersion & 0.437 & 0.437 & $\mathbf{0 . 7 3 6}$ & & \\
Fun & 0.094 & 0.363 & 0.264 & $\mathbf{0 . 7 1 5}$ & \\
Autonomy & 0.308 & 0.526 & 0.573 & 0.407 & $\mathbf{0 . 7 7 5}$
\end{tabular}

\section{Discussions and Conclusion}

Ryan, Rigby and Przybylski ([38]) have argued that gameplay is intrinsically motivating mainly because games are able to facilitate psychological need satisfaction of autonomy, competence and relatedness. According to SDT, competence is connected to optimal challenges, intuitive controls and positive feedback which includes often rewards and achievements. Gameplay engenders experiences of autonomy since playing is voluntary activity and because the player can experience to be the locus of causality in relation to in-game events. Relatedness is connected to multiplayer-situations, and possibly also to experiences of interacting with artificial intelligence in gameplay.

This study was not initially based on the SelfDetermination theory. SDT-based theoretical considerations were introduced, because the results of both of the EFAs (Study 1, Study 2) encouraged a SDT-based interpretation. The results of this study are however largely congenial with SDT theoretical argumentation on human motivations. Based on two EFAs and the CFA, it can be argued that players play digital games because of five fundamental reasons: they wish to experience Relatedness, Competence, Autonomy, Fun, and Immersion.

However, the validated model (Study 3) is also partly incompatible with the SDT. It cannot be concluded based on results of this study that Fun and Immersion are motivating gameplay experience types only because they arguably result from need satisfaction of Autonomy, Relatedness, and Competence. Rather, the results of our quantitative analyses indicate that players in Japan, Finland, and Canada consider experiences of Fun, Immersion,
Autonomy, Relatedness and Competence to be related yet separable from each other.

The IMG model does not explicitly take into account that players may be motivated e.g. by visual aesthetics in games. This is because player preferences in activity types, challenge types, as well as in narrative, interactive, and audiovisual qualities of games are considered in our approach as a research subject for gameplay type preference studies. That is to say that although many gamers find story elements of games attractive ([6][46]), an indeed continue to play a game to reveal its story, more profound general motivations may underlie also this attraction.

The validated 15-item IMG inventory is a psychometrically sound instrument for investigating intrinsic motivations to gameplay. As we specified in Study 1, items which describe players' desire to avoid boredom or anxiousness were excluded from the scale development. This is a limitation which should be addressed in future research.

The correlations (Figure 1) between the SDT need-based motivations and Fun and Immersion were all strong $(>.60)$ or very strong in our study, with the exception of the correlation between Relatedness and Fun. Future research should investigate further how SDT need based motivations to play and motivations to experience fun and immersion are related, and what can be learned by studying these relationships. For instance, it could be investigated whether the SDT need based motivations can be regarded firstorder motivations and Fun and Immersion as secondorder motivations to play. Such a study could ask to which extent players' reflections on playing because of Fun and Immersion are explained by their motivations to play because of Autonomy, Competence, and Relatedness. Perhaps by referring to 
gaming as a fun experience, players think back also on how they felt competent, autonomous, and related to the others while playing. It could also be further analyzed if Immersion and Fun mediate the effect between SDT need satisfaction and e.g. gameplay enjoyment and appreciation ([33][41][42][46]).

SDT theorists ([38]) have developed the Player Experience of Needs Satisfaction (PENS) scale which measures players' in-game satisfaction of autonomy, competence, and relatedness. The PENS has been applied e.g. in online surveys in which respondents have been asked to think their favorite game and to report how they felt during gameplay ([31]). In future research, a survey with both the IMG and the PENS could be designed to study how players' motivations to play are related to how their psychological needs were satisfied in their favorite gameplay experience. By doing so, both construct and discriminant validity for the IMG could be further investigated.

Next step in the development of the IMG model is to relate it to other three main categories of player preference research (player behavior, gaming intensity, gameplay type preferences). In future research, the CFA measurement model can be utilized in also predicting e.g. expenditure of time and money on games, as well as in studies to other subjects which investigate media effects, or e.g. economic, social, and cultural capital ([25]).

\section{Acknowledgements}

This work has been supported by Business Finland (5479/31/2017, 864/31/2016, 40009/16) and participating partners, Satakunnan korkeakoulusäätiö and its collaborators, and Academy of Finland (Center of Excellence in Game Culture Studies).

\section{References}

[1] R. A. Bartle, "Hearts, Clubs, Diamonds, Spades: Players Who Suit MUDS.", www.mud.co.uk/richard/hcds.htm, 1996.

[2] R. A. Bartle, Designing Virtual Worlds, New Riders, Boston, 2003.

[3] C. Bateman, R. Lowenhaupt and L. E. Nacke, "Player typology in theory and practice.", Proceedings of DiGRA 2011 Conference: Think Design Play, 2011, Tampere, Finland, pp. 1-13.

[4] T. A. Brown, Confirmatory Factor Analysis for Applied Research. Second Edition, The Guilford Press, New York, 2015.

[5] B. Cowley and D. Charles, "Behavlets: a method for practical player modelling using psychology based player traits and domain specific features.", User Modeling UserAdapted Interaction 26(2), 2016, pp. 257-306.
[6] F. De Grove, V. Cauberghe, and J. Van Looy, "Development and Validation of an Instrument for Measuring Individual Motives for Playing Digital Games.", Media Psychology 19, 2016, pp. 101-125.

[7] F. De Grove, J. Breuer, V.H.H. Chen, T. Quant, R. Rabindra and J. Van Looy, "Validating the Digital Games Motivation Scale for Comparative Research Between Countries.", Communication Research Reports 34(1), 2017, pp. 37-47.

[8] E. L. Deci and R. M. Ryan, Intrinsic motivation and self-determination in human behavior. Plenum, New York, 1985.

[9] S. Deterding, Modes of Play. A Frame Analytic Account of Video Game Play. Academic Dissertation, University of Hamburg, Hamburg, 2013.

[10] A. M. Farrell, "Insufficient discriminant validity: a comment on Bove, Per-van, Beatty and Shiu.", Journal of Business Research, 63(3), 2009, pp. 324-327.

[11] C. Fornell and D. F. Larcker,"Evaluating structural equation models with unobservable variables and measurement error.", Journal of Marketing Research, 18(1), 1981, 39-50.

[12] E. Goffman, Frame Analysis. An Essay on the Organization of Experience. Northeastern University Press, Boston, 1986 [1974].

[13] B. S. Greenberg, J. Sherry, K. Lachlan, K. Lucas and A. Holmstrom, "Orientations to video games among gender and age groups.", Simulation and Gaming, 41(2), 2010, pp. $238-259$

[14] M. D. Griffiths, M. N. O. Davies, and D. Chappell, "Breaking the stereotype: The case of online gaming.", CyberPsychology and Behavior, 6(1), 2003, pp. 81-91.

[15] M. D. Griffiths, M. N. O. Davies, and D. Chappell, "Demographic factors and playing variables in online computer gaming.", Cyberpsychology and Behavior, 7(4), 2004, pp. 479-487.

[16] J. Hamari and L. Keronen, "Why do people play games? A Meta-Analysis.", International Journal of Information Management, 37(3), 2017, pp. 125-141.

[17] J. Hamari and J. Tuunanen, "Player types: A metasynthesis.", Transactions of the Digital Games Research Association, 1(2), 2014, pp. 29-53.

[18] R. K. Henson and J. K. Roberts, "Use of exploratory factor analysis in published research: Common errors and some comment on improved practice.", Educational and Psychological Measurement, 66, 2006, pp. 393-416.

[19] L. T. Hu and P. M. Bentler, "Cut off criteria for tindexes in covariance structure analysis: Conventional criteria versus new alternatives.", Structural Equation Modeling: A Multidisciplinary Journal, 6(1), 1999, pp. 155.

[20] B. Ip and G. Jacobs, "Segmentation of the games market using multivariateanalysis.", Journal of Targeting, Measurement and Analysis for Marketing, 13(3), 2005, pp. 275-287.

[21] A. S. Kahn, C. Shen, L. Lu, R.A. Ratan, S. Coary, J. Hou, J. Meng, J. Osborn and D. Williams, "The Trojan Player Typology: A cross-genre, cross-cultural, behaviorally validated scale of video game play motivations.", Computers in Human Behavior 49, 2015, pp. 354-361. 
[22] K. P. Kallio, F. Mäyrä and K. Kaipainen. "At least nine ways to play: approaching gamer mentalities", Games \& Culture, (6)4, 2011, pp. 327-353.

[23] R. B. Kline, Principles and Practice of Structural Equation Modeling. Third Edition, The Guilford Press, New York, 2010.

[24] J. Koivisto and J. Hamari, "Demographic differences in perceived benefits from gamification.", Computers in Human Behavior, 35, 2014, pp.179-188.

[25] H. Korkeila, J. Hamari, "The relationship between player's gaming orientation and avatar's capital: A study in Final Fantasy XIV.", In Proceedings of the 51st Hawaii International Conference on System Sciences, Hawaii, USA, 2018, pp. 1893-1902.

[26] M. Krcmar and Y. Strizhakova, "Uses and gratifications as media choice". In: T. Hartmann (ed.), Media Choice: A Theoretical and Empirical Overview, Routledge, New York, 2009, pp. 53-69.

[27] H.W. Marsh and K. T. Hau, Z. Wen, "In search of golden rules: Comment on hypothesis-testing approaches to setting cut off values for t-indexes and dangers in overgeneralizing $\mathrm{Hu}$ and Bentler's (1999) findings.", Structural Equation Modeling 11(3), 2004, pp. 320-341.

[28] M. Matsunaga, "How to Factor-Analyze Your Data Right: Do's, Don'ts, and How-To's.", International Journal of Psychological Research, 3(1), 2010, pp. 97-110.

[29] A. W. Meade and S. B. Craig, "Identifying Careless Responses in Survey Data. Psychological Methods.", Advance online publication, 2012.

[30] J. Mulligan and B. Patrovsky, Developing Online Games: An Insider's Guide, San Francisco, New Riders, 2003.

[31] J. L. D. Neys, J. Jansz and E. S. H. Tan, "Exploring persistence in gaming: The role of self-determination and social identity.", Computers in Human Behavior 37, 2014, pp. 196-209.

[32] J. Piaget, Play, Dreams and Imitation in Childhood, W. W. Norton \& Company, Inc, New York, 1962 [1951].

[33] A. K. Przybylski, R. Ryan and C. S. Rigby, "The Motivating Role of Violence in Video Games.", Personality and Social Psychology Bulletin, 35(2), 2009, pp. 3-259.

[34] A. Przybylski, C. S. Rigby and R. M. Ryan, "A Motivational Model of Video Game Engagement. Review of General Psychology.", 14(2), 2010, pp.154-166.

[35] T. Quandt, H. Gruninger and J. Wimmer, "The grey haired gaming generation: Findings from an explorative interview study on older computer gamers.", Games and Culture 4, 2009, pp. 27-46.

[36] D. W. Russell, "In search of underlying dimensions: The use (and abuse) of factor analysis in Personality and Social Psychology Bulletin.", Personality and Social Psychology Bulletin, 28, 2002, pp. 1629-1646.

[37] R. Ryan and L. Deci, "Intrinsic and Extrinsic Motivations: Classic Definitions and New Directions. Contemporary Educational Psychology.", 25(1), 2000, pp. 54-67.

[38] R. M. Ryan, C. S. Rigby and A. Przybylski. The Motivational Pull of Video Games: A Self-Determination Theory Approach.", Motivation and Emotion, 30 (4), 2006, pp. 344-360.
[39] J. B. Schreiber, A. Nora, F. K. Stage, E. A. Barlow and J. King, "Reporting structural equation modeling and confirmatory factor analysis results: A review.", The Journal of Educational Research, 99, 2006, pp. 323-337.

[40] J. L. Sherry, K. Lucas, B. S. Greenberg \& K. Lachlan, "Video game uses and gratifications as predictors of use and game preference.", In P. Vorderer and J. Bryant (Eds.), Playing Video Games. Motives, Responses, and Consequences, Routledge, Taylor \& Francis Group, New York, 2006, pp. 213-224.

[41] R. Tamborini, N. D. Bowman, A. Eden, M. Grizzard and A. Organ, "Defining Media Enjoyment as the Satisfaction of Intrinsic Needs.", Journal of Communication, 60, 2010, pp. 758-777.

[42] R. Tamborini, M. Grizzard, N. D. Bowman, L. Reinecke, R. J. Lewis and A. Eden, "Median Enjoyment as Need Satisfaction: The Contribution of Hedonic and Nonhedonic Needs.", Journal of Communication, 60, 2011, pp. 1025-1042.

[43] G. F. Tondello, R. R. Wehbe, R. Orji, G. Ribeiro and L. E. Nacke, "A framework and taxonomy of videogame playing preferences.", In Proceedings of the Annual Symposium on Computer-Human Interaction in Play, 2017, pp. 329-340 (2017).

[44] G. F. Tondello, D. Valtchanov, A. Reetz, R. R. Wehbe, R. Orji and L. E. Nacke, "Towards a Trait Model of Video Game Preferences.", International Journal of Human-Computer Interaction, 2018.

[45] F-C. Tseng, "Segmenting online gamers by motivation.", Expert Systems with Applications, 38, 2010, pp. 7693-7697.

[46] J. Vahlo, In Gameplay. The Invariant Structures and Varieties of the Video Game Gameplay Experience. Academic Dissertation, University of Turku, Turku, 2018.

[47] J. Vahlo, J. Kaakinen, S. Holm and A. Koponen, "Digital game dynamics preferences and player types.", Journal of Computer-Mediated Communication, 22(2), 2017, pp. 88-103.

[48] J. Vahlo and A. Koponen, "Player personas and game choice.", In N. Lee (ed.), Encyclopedia of Computer Graphics and Games, Springer International Publishing, Cham, Switzerland, 2018.

[49] J. G. Webster, The Marketplace of Attention. How Audiences Take Shape in a Digital Age, The MIT Press, Cambridge, 2014.

[50] D. Williams, N. Yee, S. Caplan, "Who plays, how much, and why? Debunking the stereotypical gamer profile.", Journal of Computer-Mediated Communication, 13(4), 2008, pp. 993-1018.

[51] N. Yee, "Motivations for Play in Online Games.", Journal of CyberPsychology and Behavior, 9, 2006, pp. $772-775$.

[52] N. Yee, N. Ducheneaut and L. Nelson, "Online Gaming Motivation Scale.", In Proceedings of the 2012 ACM annual conference on Human Factors in Computing Systems ACM 2012, Austin, USA, 5-10, May 2012, pp. 2803-2806.

[53] A. Zait, P. Bertea, "Methods for Testing Discriminant Validity.", Management \& Marketing, IX (2), 2011, pp. 217-224. 